\title{
Subject Review: Video Compression Algorithms
}

\author{
Amal Abbas Kadhim ${ }^{1}$, Azal Minshed Abid ${ }^{2}$ and Zuhair Hussein Ali ${ }^{3}$ \\ ${ }^{1-3}$ Department of Computer Science, College of Education \\ Mustansiriyah University
}

Iraq.

\begin{abstract}
Video compression is the process of decreasing the number of bits required to represent a certain video. Video compression can be done by a specific algorithm for deciding the most ideal approach to reduce the amount of data storage. The video file is coded in such a way that consuming less space than the original file and is easier to transmit over the Internet. The basic idea of video compression based on removing the redundant data that exists in the video. There are four types of redundancy in digital video: colorize redundancy, temporal redundancy, statistical redundancy, and spatial redundancy. Video compression algorithms must reduce these redundancies in such a way that keep the quality of the compressed video when the decompression process is done. Most video compression techniques consist of the following steps: Motion Estimation,Motion Compensation, Discrete Cosine Transform, Run Length Encoding, Huffman Coding. Frame Difference. This paper discusses the characteristic of video coding from the scratch of key frame selection to the evolutions of various standards
\end{abstract}

Key Words:Video compression, PSNR, Frame, Spatial redundancy, Discrete Cosine Transform.

\section{INTRODUCTION}

Digital video can be defined as a number of images called frames displayed in a specific sequence with a fixed time between each image to be as a motion images. There are a few changes between frames that we can't observe it when video displayed. Digital video used widely in many applications such as learning, communication and broadcasting. The significant test for productive advanced video storage and transmission lies in the enormous measure of information expected to show computerized video, and thus huge memory space is needed to store video pictures, and similarly huge data transfer capacity for their transmission, so video compression used to deal with such problems of video transmission and the need of high memory space. Digital video compression is the way toward encoding a video file so that it occupies less space than the original video and is simpler to communicate over the Internet. The main idea of video compression based on reducing the size of video file formats by eliminating redundant and non-functional data from the original video file. Many algorithms used for video compression that based on eliminating repetitive images in the video, For instance, a video may have a similar background, picture or sound played a few times or the information showed with video document isn't excessively significant. Video compression will eliminate all such information to decrease the video size. Video compression may lead to change the format of the compressed video to another format based on the compression algorithm used, so video player must be supported to enable the video to play. There are four types of redundancy in digital video: colorize redundancy, temporal redundancy, statistical redundancy, and spatial redundancy. These redundancies are not similar where each one of them has different characteristics[1]. There are two parts for any video compression system: video encoder and video decoder. Video encoder responsible for converting the original video format to another video format for the purpose of compression and an efficient transmitting of video, While video decoder responsible for decompressed video and back to the original format. There are three functions in the encoder part that enable to, remove video redundancy: color subsampling, inter-frame encoder and intra-frame encoder[2]. Efficient compression can be measured by the inter-frame encoder. The inter-frame is an important frame that can express the neighbor frame, also can be used effectively in frame prediction that is necessary for video compression. Temporal redundancy can be reduced using inter-frame that help to discover the correlation between sequential frames, which is useful for reducing temporal redundancy. High compression can be achieved by frame prediction that help to reduce temporal redundancy so the transmission rate of video frame decreased[3]. Video compression can be lossy or lossless. For lossy compression the objective is to improve compression methods that are proficient and bring about perceptually lossless quality. Essentially, despite the fact that the compressed video is not quite the same as the first uncompressed video when decompressed video applied, the distinctions are not effectively noticeable to the natural eye, while the lossless video compression based on strategy that the compressed video is the same as the 
uncompressed video and the original video can be retrieve without any change[4]. Figure 1 shows the block diagram of video compression. In this paper many video compression techniques are investigated according to its chronological order

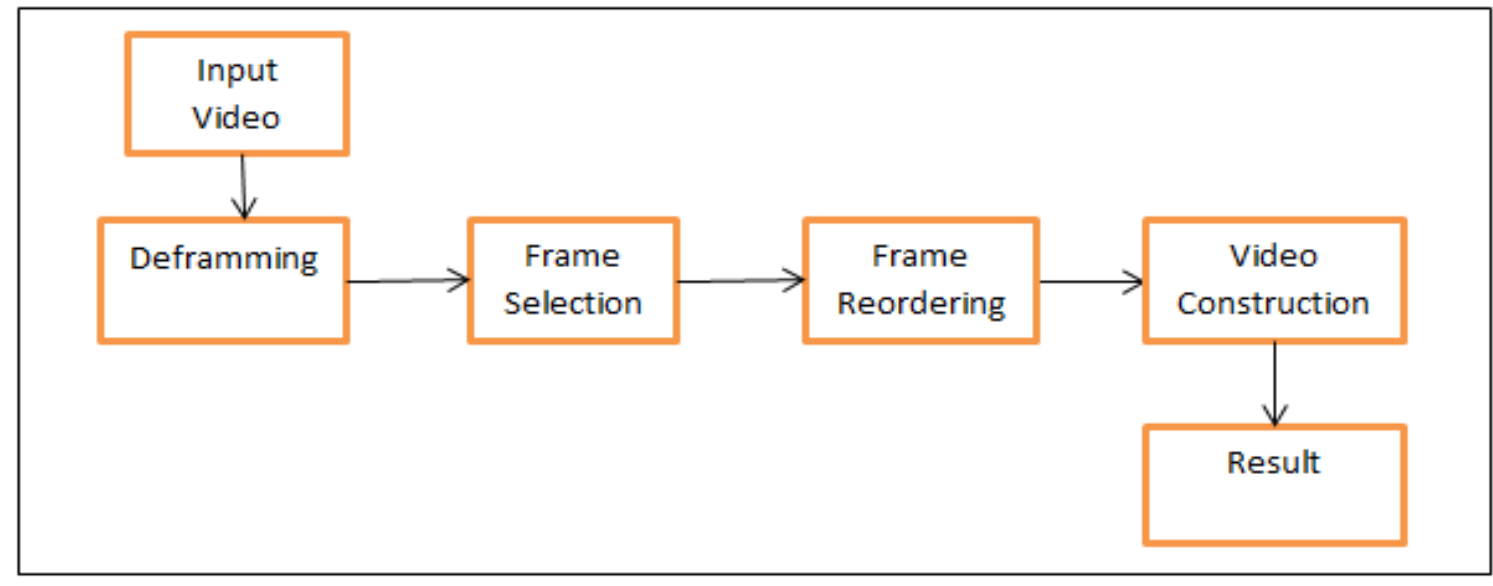

\section{Video Compression Techniques}

Figure 1: Block Diagram of Video Compression

Digital video contains a huge amount of data that required large bandwidth and storage capacity when transmitting it. For these reasons video compression take research attention to develop new and efficient techniques for video compression, these techniques based on saving the changes between frame pixels only and the same pixels that do not change between successive frames save only one time. Below are some of most important video compression techniques.

\subsection{H-120}

It was produced by ITU-T at 1984 as the first video compression technique. H,120 working at $1544 \mathrm{kbit} / \mathrm{s}$ for NTSC and $2028 \mathrm{kbit} / \mathrm{s}$ for PAL. This Type of compression provided less video quality, although H.120 represent the first standard coding for H.xxx family it has few implementations Due to its weak efficiency. The second version of H.120 introduced in 1988 which added motion compensation and background predication. As most video compression techniques H.120 used discrete cosine transform and run length[5].

\section{$2.2 \mathrm{H}-261$}

Developed by ITU-T in 1988 for standard video compression. The objective is to create a hybrid method for video compression that is the basis for many compression techniques and enable video to be transfer by ISDN lines through multiple of $64 \mathrm{kbit} / \mathrm{s}$ data rate. There are two methods in the hybrid video coding technique, First the movement from frame to frame is estimated and remunerated for by predicating frame from the earlier coded frame. Second The differences between specific frame and predicted frame is decoded by computing the correlation between them. Due to it's efficiency in video coding $\mathrm{H}-261 \mathrm{used}$ widely in the video-conference and also used in a teleconference. There are three main elements for H-261 : prediction, transformation of block from spatial domain to the frequency domain using Discrete Cosine Transform(DCT) and entropy coding[6].

\subsection{MPEG-1}

Moving Picture Experts Group(MPEG-1) standard was introduced in 1993 by ISO/IEC. MPEG-1 can be playable in all PC's, VCD player and DVD players, also can be used for both video and audio. MPEG-1 video can be viewed as a series of images. These images are, in turn, represented using a two-dimensional array of pixels. There are three components for each pixel color : one luminance and two chrominance. Spatial redundancy and temporal redundancy can be reduced in a MPEG-1 using hybrid method that enables to reach high compression ratio. There are many steps for MPEG-1 include: color space conversion and subsampling of chrominance information, quantization, discrete cosine transform, Zig-Zag Scan and run length encoding, predictive coding and motion estimation. MPEG-1 suffers from lack of support interlaced pictures, that lose the ability to run PAL and NTSC video format that based on interlaced pictures, which helped to develop MPEG-2 that supports interlaced pictures [7]. 


\subsection{MPEG-2}

Developed by ISO and ITU in 1995 to support audio and video. Which includes the integration of the ability to deal with lossy video and lossy audio, which helps in the transfer and storage of movies through available media in a good way. Despite its inefficiency compared to H.264 and H.265, it is still widely used. MPEG-2 is generally utilized as the organization of TV signals that are communicated by earthbound, link, and direct transmission satellite TV frameworks. It likewise determines the configuration of movies and different programs that are spread on DVD and comparable discs. Television slots, TV recipients, DVD players, and other equipment are frequently intended to this model. There are two main parts to MPEG-2 that are different, but their work is related to each other: transport stream and program stream. Transport stream includes M2TS, AVCHD and HDV, where M2TS utilized in Blue-ray discs, while AVCHD and HDV used in re-writable DVD and compact flash cards respectively. Program stream includes VOB and Enhanced VOB. As As mentioned earlier in this section MPEG-2 can used for both video and audio. Video in MPEG-2 is similar to MPEG-1 but it supports interlaced pictures. . MPEG-2 video is not suitable for low bit-rate, mainly less than $1 \mathrm{Mbit} / \mathrm{s}$. MPEG-2 supports audio encoding that not available in MPEG-1. MPEG-2 can be used to support three pictures type: intra $(\mathrm{I})$ coded without reference to other frames, Predicted $(\mathrm{P})$ - coded depend on prediction from prior $\mathrm{I}$ or $\mathrm{P}$ frame, and Bidirectionally predicted (B) — predicted from P and or I frames but not used as a basis for further predictions[8].

\subsection{H.263}

Depending of H.261 and the efficiency in it H.263 has been developed. The H.263 standard (1996) created by the ITU Its main objective was to hold videoconferencing at low cost and high efficiency. Particularly for cumulative video. The quality of H.263 is better than all previous video compression techniques at bit rate. H.263 provide better video quality compared to MPEG-2 and H.261 In terms of storage and transportation. H.120 uses DCT for the transformation that decreases the entropy and increases the performance of the encoder where the Huffman and arithmetic coding for coding the input. The using of DCT allowed the exploited of perceptual properties [9].

\subsection{MPEG-4}

MPEG-4 developed by ISO/IEC in 1999 it a hybrid method for compression of both video and audio. Many features of MPEG-1 and MPEG-2 included in MPEG-4 with some new features such as VRML support for 3D rendering object-oriented composite files and various types of interactivity. MPEG-4 provides many facilities to the developers So that they can add things that develop the coding process. Some of these facilities allow developer to add many software and hardware to improve the quality of provided services. The transmitted data can be converted to another signal that compatible with any available network. The end users provided with a large range of interaction. There are many pros of MPEG-4 some of them:provide high compression ratio compared to other standards, also can can contain metadata and extensible meta data platform, The file can be integrated with 3D graphics menus, There are many open source players that can support this format and it is versatile file that can support audio or video in easy way. The limitation of MPEG-4 are the format is contained, that instead of storing the ode of the file the data is stored that no unique code for all files also there are many codecs that lead to many kinds of players that play specific code and can not play all codecs[10].

\subsection{H.264}

Provided by ISO/IEC with ITU-T in 2003 also called MPEG-4 part 10 is a standard video compression based on block oriented. The H.264 video standard is a H.26x family standard defined by ITU-T. Its development is a joint work of the MPEG and VCEG teams, which originally supported by ISO and ITU-T organizations, respectively. In the past, these two groups have jointly done MPEG-2 or H.262. This standard is sometimes called JVT (Joint Video Team), after the team that developed it. In the MPEG framework, the symbol AVC (Advanced Video Coding) was chosen by analogy with the AAC codec of the MPEG-2 Part 7 audio standard, which was also named to distinguish it from the popular MPEG-2 Part 3 audio codec (MP3). The motivation behind making H.264/AVC was to pioneer another digital video standard fit for conveying great video quality at a substantially lower bit rate than prior standards at a significantly lower bitrate than previous standards. H,264 is flexible so it can be used in many applications. H.264 provides many facilities including: provided smaller file size that is less three times than the file provided by MPEG-2 making this compression more efficient than others. Suitable for slow motion video content, high quality video content, provide real time video transmission, and reduces the size of a digital video size to be stored. Some limitation also associated with using H.264: has a sophisticated and complicated video format making it difficult to be understood, use of H.265 can produce inconsistent image quality, and it It needs more hardware overhead as compared to other video compression $\operatorname{codec}[11]$.

\section{$2.8 \quad H .265$}

Introduced by ISO/IEC with ITU-T in 2013. HEVC (H.265) high-quality video codec is the successor to the H.264 codec, which was specially developed with H.264 encoding by efforts both ISO/IEC and ITU-T. The compression ratio of H.265 is better than the compression ratio of $\mathrm{H} .264$ with rate up to $50 \%$, so it is used widely in various applications . H.265 is a 
long line of video compression standards. Like its predecessor, H. ... 264 is for SD and HD resolutions, its ability to evolve to handle the demands of the latest high-resolution. H.265 deal with a block of size $64 * 64$ pixel that provide high quality pictures. H.265 provide a good bit-stream because it combines the original technologies with advanced techniques, Better improvements can be obtained by balancing between time delay and algorithm complexity to enhance the improvement of coding quality I [12].

\section{Quality Measure in Video Coding}

In video compression, lossy methodology is the primary strategy used to accomplish a great compression proportion; be that as it may, this methodology prompts lost data after retrieving of the original video. So as to evaluate the nature of the recreated video, a few strategies have been created. One of the least difficult and most mainstream techniques is to utilize Mean Square Error (MSE) for each frame independently. The MSE can be computed as in Eq.(1).

$$
\operatorname{MSE}=\frac{1}{M * N} \sum_{i=1}^{M} \sum_{J=1}^{N}\left(f(i, j)-F^{\prime}(i, j)\right)^{2}
$$

Where

$\mathrm{M}$ and $\mathrm{N}$ are the frame size

$\mathrm{F}(\mathrm{i}, \mathrm{j})$ is the original pixel value.

$F^{\prime}(i, j)$ is the reconstructed pixel value.

Also Peak Signal to Noise Ratio(PSNR) can be used to compute the quality of the compressed video . PSNR is mostly utilized as a video quality measurement it offers a good correlation with subjective data. The PSNR can be computed as in Eq. (2).

Where

$$
\operatorname{PSNR}(\mathrm{i})=10 \log _{10} \frac{\mathrm{I}}{\operatorname{MSE}(\mathrm{i})}
$$

I is the maximum pixel luminance value (e.g. 255 for 8 -bit representation).

The quality can be measured by computing the PSNR between the original video and the reconstructed video. The PSNR is computed for each frame in the video separately. High PSNR refers to high quality while low PSNR refers to low quality[1]

\section{CONCLUSIONS}

This paper presents a detailed review of many approaches of video compression techniques to support the reduction of space complexity and network overhead. Video compression is ending up more notable considering the way that capacity and system transmission bandwidth necessities can be reduced by compression. The main goals of the various video compression techniques are achieving a high compression ratio, transmission capacity adaptability and high quality video when decompression process is done.

\section{REFERENCES}

1. Hussain,A. \& Ahmed,Z .(2018). A Survey on Video Compression Fast Block

Matching Algorithms, Neurocomputing, doi: https://doi.org/10.1016/j.neucom.2018.10.060

2. BOVIK, A. C. (2009). "Chapter 1 - Introduction to Digital Video Processing". In:The Essential Guide to Video Processing, 2nd edition; Boston: Academic Press.

3. Bahari, A., Arslan, T., \& Erdogan, A. T. (2010). Interframe Bus Encoding Technique and Architecture for MPEG4 AVC/H.264 Video Compression, IEEE Transactions on Very Large Scale Integration (VLSI) Systems, DOI: 10.1109/TVLSI.2009.2015324.

4. Abedi, M.; Sun, B.; Zheng, Z. (July 2019). "A Sinusoidal-Hyperbolic Family of Transforms With Potential Applications in Compressive Sensing". IEEE Transactions on Image Processing. 28 (7): 3571-3583.

5. Abedi, M., Sun, B. \& Zheng, Z. (July 2019). A Sinusoidal-Hyperbolic Family of Transforms With Potential Applications in Compressive Sensing. IEEE Transactions on Image Processing. 28 (7): 3571-3583 
6. Haseeb, S. \& O.O. Khalifa,( 2006). Comparative performance analysis of image compression by JPEG 2000: A case study on medical images. Inform. Technol. J., 5: 35-39.

7. Mitchell,J.L., Pennebaker,W.,B., Fogg,C.,E. \& LeGall,D.,J,(1996), "MPEG Video Compression Standard," Digital Multimedia Standards Series, Chapman and Hall, London, pp. 135-169.

8. Rajwant, K. \& Anshu, B.(2018), A Recent Survey of Different Video Compression Methods, International Journal of IT \& Knowledge Management, Vol. 11 , NO. 2 pp. 103-109

9. Nilsson, M. \& M. Naylor,( 2003). Comparison of H.263 and H.26L video compression performance with web-cams. Electronics Lett., 39: 277-278.

10. Iain E. G. (2002), Video Coding Standards: H.261, H.263 and H.26L, John Wiley \& Sons.

11. SUlLiVAN, G., TOPIWALA, P. \& LUTHRA, A. (2004). "The H.264/AVC Advanced Video Coding Standard: Overview and Introduction to the Fidelity Range Extensions". SPIE conference on Applications of Digital Image Processing XXVII.

12. Miroslav, U., Jaroslav,F., Lukas, S. \& Martin ,V.(2014), Impact of H.264/AVC and H.265/HEVC Compression Standards on the Video Quality for 4K resolution, DIGITAL IMAGE PROCESSING AND COMPUTER GRAPHICS,vol. 12 ,No.14 